\title{
Analysis of the Performance of Decentralized Sensor Network with Correlated Observations
}

\author{
Nithya Gnanapandithan and Balasubramaniam Natarajan \\ WiCom Group \\ Department of Electrical and Computer Engineering \\ Kansas State University \\ Manhattan, Kansas 66506-5204 \\ nithyag@ksu.edu,bala@ksu.edu
}

\begin{abstract}
In this paper, we study the performance of a decentralized sensor network in the presence of correlated additive Gaussian noise. We propose a Parallel Genetic Algorithm approach to simultaneously optimize both the fusion rule and the local decision rules in the sense of minimizing the probability of error. Our results show that the algorithm converges to a majority-like fusion rule irrespective of the degree of correlation and that the local decision rules play a key role in determining the performance of the overall system in the case of correlated observations. We also show that the performance of the system degrades with increase in the correlation between the observations.
\end{abstract}

\section{INTRODUCTION}

Decentralized processing, wherein the local sensors perform some preliminary processing of data and then send the compressed information to a central processor (fusion center), has the advantages of reduced communication bandwidth requirement, reduced cost and increased reliability. The fundamental problem in decentralized processing is to optimize the performance of the system with respect to the probability of detection at the fusion center by determining the optimal local and global decision rules. This problem has been studied extensively based on the assumption that the observations of the local sensors are conditionally independent when conditioned on the hypothesis [1], [2]. This assumption simplifies the problem and makes it more tractable since, in this case, the optimal local classifiers are likelihood ratio tests characterized by a finite number of thresholds [4]. However, this assumption of conditional independence is not always valid in practice [7]. This is intuitively true in cases where the physical proximities of the local sensors to each other results in the noise on each sensor being dependent. Hence, there is a need to investigate the problem of distributed detection with correlated sensor observations.

The analysis of different detector structures in the presence of dependent noise has been carried out for centralized detection scenario [5], [6]. In [7], Lauer and Sandell studied the problem of distributed detection in presence of correlated Gaussian noise and derived suboptimum decision rules based on likelihood ratio tests. Aalo and Vishwanathan considered a similar problem in [8] assuming that the local sensors make binary decisions with all of them operating at the same threshold. Their study involved the evaluation of the probability of detection in the Neyman-Pearson (N-P) sense when the fusion rule was fixed to be one of the standard rules such as AND, OR or Majority Voting rule. In [9], Drakopolous and Lee derived the optimum decision rule in the N-P sense when the local decision rules and the correlations between the local observations are given. Aalo and Vishwanathan also studied the asymptotic performances of distributed and centralized detection systems in the presence of correlated Gaussian noise in [10]. In [11], the optimal fusion rule is developed for correlated local binary decisions by using the Bahadur-Lazarsfeld expansion of probability density functions. Thus, most of the literature has been devoted to deriving the optimal fusion rule for a given set of local decision rules or vice versa. The problem of simultaneously optimizing both the fusion and local decision rules has not been tackled for the dependent noise case.

In this paper, we provide a Parallel Genetic Algorithm (PGA) approach for optimizing both the local and global decision rules simultaneously. We analyze the performance of distributed sensor networks in the presence of correlated Gaussian noise for the case when the local classifiers are assumed to be likelihood ratio tests characterized by a finite number of thresholds. Unlike most of the prior work in this field, we consider non-binary local decision rules. As in [8], we consider both positively and negatively correlated symmetric multidimensional noise distributions which can be completely characterized by a single correlation coefficient $\rho$. However, our approach can be extended for any arbitrary positive definite noise covariance matrix structure. Furthermore, we present the results for the case when the sensor thresholds are non-homogeneous (non-identical) as well as for the homogeneous (identical) case. Our results show that the performance of the decentralized network degrades as the correlation between the sensors increases. This is intuitively correct as we would expect the distributed system to become equivalent to the single sensor system when the correlation coefficient is equal to 1 . In addition to presenting all these results for the two sensor case, we also show the convergence of our algorithm for the three sensor case. Our results show that the algorithm converges to a majority-like fusion rule for all the cases irrespective of the degree of correlation. The local decision rules, on the other hand, are different for the different 
cases. Thus, it is the local decision rules that play a major role in determining the performance of the decentralized sensor network when the sensor observations are correlated. The PGA approach proposed in this paper is effective in determining the optimal local decision rules.

The rest of the paper is organized as follows. In section II, we provide the system model and describe the problem at hand. We present our Parallel Genetic Algorithm in section III. In section IV, we present our results, and in section V we give our conclusions.

\section{SySTEM MODEL}

Consider the parallel decentralized detection system shown in Fig.1, where the $N$ local sensors gather observations $y_{n}$, make a local decision $u_{n}$ per sensor, and transmit these decisions to a single fusion rule $\gamma_{0}$ through an error-free multiple access channel.

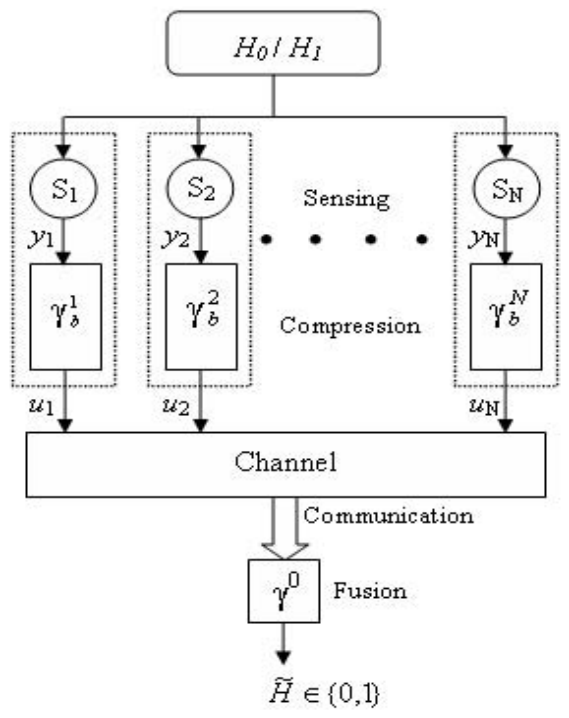

Fig. 1. Parallel fusion network

We consider the binary detection problem in such a system, (i.e.) we are testing the two hypotheses $H_{1}$ (signal present) and $H_{0}$ (no signal). The two hypotheses have prior probabilities $\pi_{1}$ and $\pi_{0}$, respectively. The observation at each sensor, $y_{i}$, is given by,

$$
y_{i}=\left\{\begin{array}{ll}
s_{i}+n_{i} & \text { under } H_{1} \\
n_{i} & \text { under } H_{0}
\end{array} \quad i=1,2, \ldots, N\right.
$$

The local detectors map these observations into one of $L=$ $2^{b}$ classes, where $b$ is the number of bits transmitted to the fusion center by each sensor. The fusion center then makes a global decision $\tilde{H}$ about the true state $H$ based on the set of local decisions from all $N$ sensors.

We assume that the noise on the sensors is additive Gaussian dependent noise. As mentioned before, we consider only symmetric noise densities which can be completely described by a single correlation coefficient. Thus, for a three sensor system, the covariance matrix for a zero mean Gaussian noise with unit variance has the following form,

$$
\Lambda=\left(\begin{array}{lll}
1 & \rho & \rho \\
\rho & 1 & \rho \\
\rho & \rho & 1
\end{array}\right)
$$

The optimum local classifiers are assumed to be likelihood ratio tests. While likelihood ratio tests have been shown to be optimal only for uncorrelated sensor observations ([3], [4]), it is still widely used as the local decision rule for the correlated observation case(see [7]-[11]). For the additive Gaussian noise case, the local sensors are assumed to be quantizers with $L$ levels, i.e., $L-1$ thresholds [2]. Thus, the local decision rule corresponds to

$$
u_{n}= \begin{cases}0 & \text { if } y_{n} \leq \lambda_{n, 1} \\ 1 & \text { if } \lambda_{n, 1} \leq y_{n} \leq \lambda_{n, 2} \\ \vdots & \vdots \\ L-1 & \text { if } y_{n}>\lambda_{n, L-1}\end{cases}
$$

where, $y_{n}$ is the local measurement at the $n$th sensor, $u_{n}$ is the corresponding local decision, and $\lambda_{n, 1}, \lambda_{n, 2}, \cdots, \lambda_{n, L-1}$, are the $L-1$ quantization thresholds of that sensor.

We represent each possible combination of local decisions by a vector of $N$ integers as follows

$$
\mathbf{u}=\left(\begin{array}{cccc}
u_{1} & u_{2} & \cdots & u_{N}
\end{array}\right), u_{n} \in\{0,1, \cdots, L-1\} .
$$

Assuming $L=2^{b}$, where $b$ is the number of bits transmitted per sensor, $\mathbf{u}$ can be represented as a string of $b N$ bits as follows

$$
\begin{array}{r}
\mathbf{u}=\left(u_{1}^{1} u_{1}^{2} \cdots u_{1}^{b} u_{2}^{1} u_{2}^{2} \cdots u_{2}^{b} \cdots u_{\mathrm{N}}^{1} u_{\mathrm{N}}^{2} \cdots u_{\mathrm{N}}^{b}\right), \\
u_{\mathrm{n}}^{j} \in\{0,1\}
\end{array}
$$

Thus, the space of all possible local decisions is spanned by a single $b N$-bit integer $q$, whose value ranges from 0 to $2^{b N}-1$. The individual values of the local decisions $u_{n}, n=$ $1,2, \cdots, N$ for a particular combination of the local decisions represented by $q$ can be extracted by using the reverse mapping function $\Psi_{n}(q)$ defined as

$$
\Psi_{n}(q)=\frac{q}{2^{b(N-n)}} \bmod L,
$$

where mod is the modulo operation and all operations are carried out in integer mode.

To represent the fusion rules, we adopt a binary representation similar to that described in [2], which accounts for the output of the fusion rule under every possible combination of the local decisions. Since there are $N$ sensors and each sensor classifies its measurement into $L$ classes, each fusion rule should account for $L^{N}$ local decision possibilities and, is therefore represented as a string of $L^{N}$ bits as follows:

$$
\begin{array}{r}
h=\left(\begin{array}{ccccc}
h_{0} & h_{1} & \cdots & h_{L^{N}-2} & h_{L^{N}-1}
\end{array}\right), \\
h_{q} \in\{0,1\}, q=0,1, \cdots, L^{N}-1
\end{array}
$$




\section{Problem Definition And the PGA Approach}

Problem Definition: Determine the optimum local thresholds and fusion rule, where optimality is defined as minimizing the probability of error at the fusion center.

The optimization of the decentralized sensor network has to be performed over all possible local thresholds and all possible fusion rules. The resulting optimization problem is NP-complete, i.e., the solution cannot be determined in polynomial time. The complexity of the problem increases exponentially with the number of sensors. Thus, an exhaustive search becomes impractical. One approach commonly used with this kind of a problem is the use of evolutionary algorithms such as genetic algorithms (GAs). A GA mimics the evolution process in biology and uses an evolution and survival-of-the-fittest mechanism to guide the search toward the fittest candidates [12].

The parallel GA that we are proposing in this paper is essentially an algorithm which optimizes both the fusion rule and the local thresholds in parallel. Each chromosome in the GA consists of two parts:

- the fusion rule, and,

- a set of local thresholds.

A random initial population is generated which consists of a fixed number of such chromosomes. The fitness of each chromosome is evaluated as the average probability of error at the fusion center for that particular combination of fusion rule and local thresholds. The average probability of error at the fusion center is given by the weighted sum of type-I and type-II errors,

$$
P_{e}(\lambda, h)=\sum_{k=0}^{1} \pi_{k} P_{k}^{0}(\bar{k}, \lambda, h)
$$

where $\pi_{k}$ is the prior probability of hypothesis $H_{k}$, $P_{k}^{0}(\bar{k}, \lambda, h)=\operatorname{Pr}\left(u_{0}=\bar{k} \mid H_{k}\right)$ is the probability of false alarm if $k=0$ or the probability of miss if $k=1$, and $\bar{k}$ is the binary NOT operation. Out of the $L^{N}$ mutually exclusive possible local decisions, we sum over those that results in $u_{0}=k$ decision at the fusion center as follows

$$
P_{e}(\lambda, h)=\sum_{k=0}^{1} \pi_{k} \sum_{\substack{q=0 \\ h_{q}=k}}^{L^{N}-1} \operatorname{Pr}\left(u_{1}=\Psi_{1}(q), \cdots, u_{N}=\Psi_{n}(q)\right)
$$

where $\operatorname{Pr}\left(u_{1}=\Psi_{1}(q), \cdots, u_{N}=\Psi_{n}(q)\right)$ is the joint probability of sensor 1 deciding $\Psi_{1}(q)$, sensor 2 deciding $\Psi_{2}(q)$, and so on. Since, the local sensors act as quantizers, this joint probability can be evaluated as the following set of multiple integrals

$$
\begin{aligned}
& \operatorname{Pr}\left(u_{1}=\Psi_{1}(q), \cdots, u_{N}=\Psi_{n}(q)\right)= \\
& \quad \int_{\lambda_{\Psi_{1}}}^{\lambda_{\Psi_{1}+1}} \cdots \int_{\lambda_{\Psi_{N}}}^{\lambda_{\Psi_{N}+1}} f_{k}\left(y_{1}, \cdots, y_{N}\right) d y_{1} \cdots d y_{N}
\end{aligned}
$$

where $f_{k}\left(y_{1}, \cdots, y_{N}\right)$ is the joint probability density of the observations $y_{1}, \cdots, y_{N}$.

After evaluating the fitness, the chromosomes undergo selection, cross-over and mutation. These processes are carried out for both parts of the chromosome in parallel. Elitism is also used to ensure that the best solutions from each generation are carried over to the subsequent generation without any mutation. Once, an offspring population is assembled with the required number of candidate solutions, the fitness is again evaluated and the whole process continues till a desired termination criterion is reached.

The initialization of the local thresholds plays a crucial part in the convergence of the algorithm. The local thresholds have to be initialized close to the region of overlap between $f_{0}(y)$ and $f_{1}(y)$ for proper convergence of the GA. This makes intuitive sense since this is the region where it would be hardest to discriminate between the two hypotheses.

\section{RESUlTS}

In this section, we present the simulation results obtained by using our PGA approach. We consider a parallel decentralized sensor network, where the global decision is made solely by one fusion center to which all the local sensors transmit their individual decisions through a error-free multiple access channel. We consider both the homogenous and heterogeneous sensor cases, i.e., the cases where the sensors have identical and non-identical thresholds, respectively. The local observations are assumed to follow the additive noise model $y=m_{i}+n$, where $m_{i}$ is the signal mean under $H_{i}, i=0,1$ and $n$ is the correlated Gaussian noise with zero-mean and symmetric covariance matrix as given in eqn (2). The signal means $m_{0}$ and $m_{1}$ are assumed to be 0 and 1 under $H_{0}$ and $H_{1}$, respectively. We only consider sensor networks consisting of 2 and 3 local sensors since, the order of integration increases with the number of sensors and increases the computational complexity. But, our approach is equally valid for larger number of sensors as well. The number of bits per sensor is assumed to be 2 . Thus, each sensor classifies its observation into one of 4 classes. The prior probability $\pi_{0}$ is 0.5 for all the cases. The simulations were run for different values of $\rho$ in the covariance matrix. The 3 local thresholds for each sensor are initialized as Gaussian distributed random values with means $-0.5,0.5$ and 1.5 , respectively. The variance of all the 3 local thresholds is set to be 0.0025 .

Fig.2 shows the convergence of the probability of error over 90 generations for different positive values of the correlation coefficient $\rho(\rho=0,0.2,0.5,0.9)$. The local sensors for this case are heterogeneous. $\rho=0$ represents the uncorrelated case, where all the sensor observations are conditionally independent when conditioned on the hypothesis $H_{i}$. For this case, the minimum global probability of error goes down to 0.248 after 70 generations. For the case where $\rho=0.2$, the minimum probability of error is 0.263 after 60 generations. Similarly, for $\rho=0.5$ and $\rho=0.9$, the minimum probability of error converges to 0.284 and 0.305 after 30 and 15 generations, respectively. From this, we can see that the probability of 


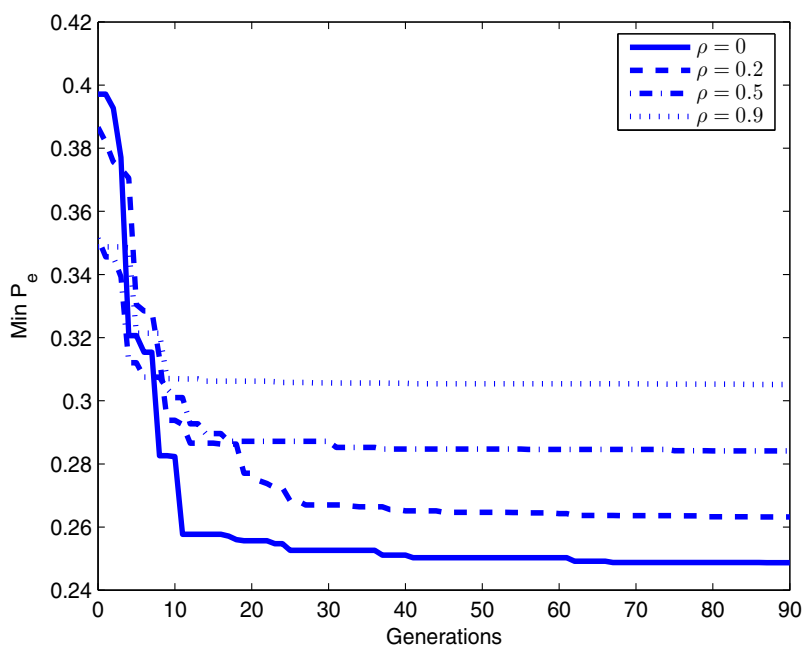

Fig. 2. Evolution of probability of error - Non-Homogenous $\operatorname{sensors}(N=$ $\left.2, L=4, \pi_{0}=0.5\right)$

error decreases with decrease in the correlation between the local sensors. This is expected as increasing $\rho$ increases the correlation among the sensor observations, thereby eventually reducing the distributed sensor network to a single sensor system as the correlation coefficient becomes 1 . Also, we note that the cases with lower value of the correlation coefficient $\rho$ take longer to converge to the optimum solution as compared to the cases with higher $\rho$ values. This is once again because the higher the correlation, the closer the network becomes to a single sensor system. The optimal local thresholds for heterogenous sensors scenario are listed in Table. I

Fig. 3 shows a similar plot for different negative correlation coefficient values over 100 generations. Once again, the sensors are heterogeneous quantizers. Here again, we can see the same trend with more negative values of $\rho$ resulting in lower probability of error. The minimum probability of error for the 3 correlated observation cases where $\rho=-0.2,-0.5$ and -0.9 goes down to $0.23,0.19$ and 0.075 , respectively. Thus, we find that there is a drastic improvement in the performance of the sensor network as the negative correlation between the observations increases. An increase in the negative correlation essentially means that if the noise on one sensor is pushes the observation towards the wrong hypothesis, then the noise on the other sensor will push it toward the correct hypothesis. Thus, if one sensor makes an erroneous decision, the chances for the other sensor making the right decision are more. The worst case would be when the magnitude of noise on both the sensors is small. In this case, the performance would be equivalent to the uncorrelated case. Thus, negative correlation on the observations would cause the network to always perform better than or at least equal to the uncorrelated case.

Fig.4 shows the convergence plot of the probability of error for the case where the sensors are all homogeneous, i.e., having the same thresholds. We find that the results are similar to the heterogeneous case. But, the algorithm is found

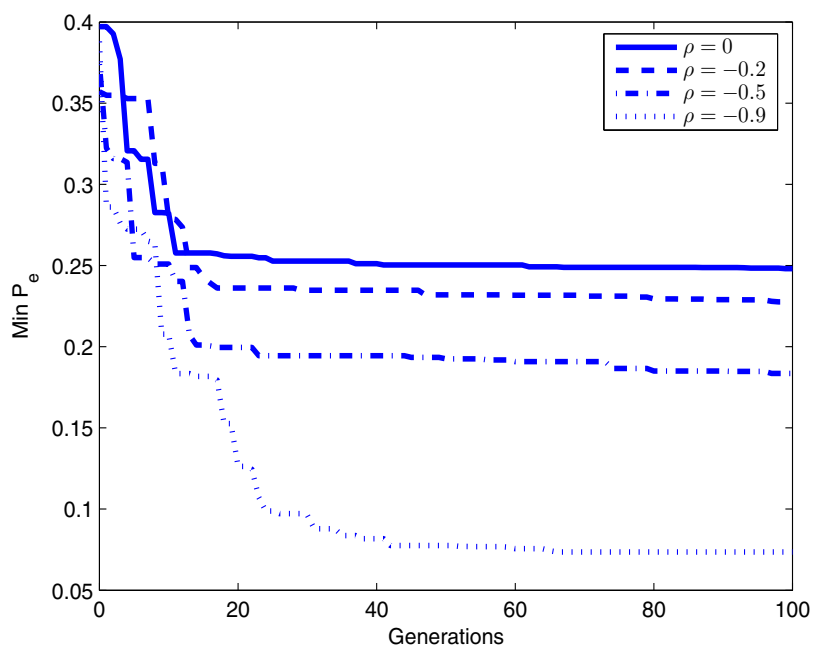

Fig. 3. Evolution of probability of error - Non-Homogenous $\operatorname{sensors}(N=$ $2, L=4, \pi_{0}=0.5$ )

to converge much faster in this case. This due to the low complexity of problem since, for the homogeneous case, we need to optimize only one set of common thresholds for the whole network instead of a set of thresholds for each sensor. The GA converges in 35 generations here as opposed to the 90 generations in the heterogeneous case. Table. II lists the local thresholds for different cases of this scenario.

Fig.5 shows the evolution of the probability of error for the 3 sensor heterogeneous case with $\rho=0.5$. The GA for this case converges after 150 generations and the minimum global probability of error at the end of 150 generations is 0.273 , which is less than the minimum probability of error for the 2 sensor case with the same value of $\rho$. Due to the computational complexity of the 3 sensor case which involves a triple integral, we only provide the results for one value of $\rho$.

The optimal fusion rule in all the above cases was found to converge to a majority-like fusion rule, where the integer sum of all the local decisions is compared to a threshold given by

$$
\lambda^{0} \simeq \frac{1}{2} N(L-1)
$$

where $N$ is the number of sensors and $L$ is the number of quantization levels per sensor. Although, all the cases converge to the same fusion rule, there is a degradation in the performance with increasing correlation. The only difference between the cases with varying degrees of correlation is in the local thresholds. Thus, we find that the local thresholds play a major role in determining the performance of the decentralized sensor network when the observations are correlated. We can see from Tables I \& II that as the correlation coefficient increases, the 3 local thresholds of each sensor drift closer to each other. 


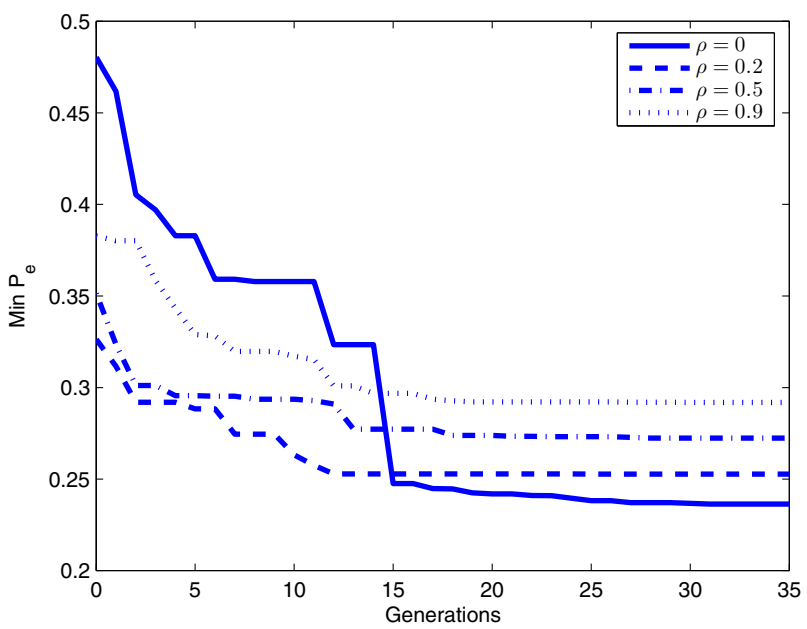

Fig. 4. Evolution of probability of error - Homogenous sensors $(N=2, L=$ $\left.4, \pi_{0}=0.5\right)$

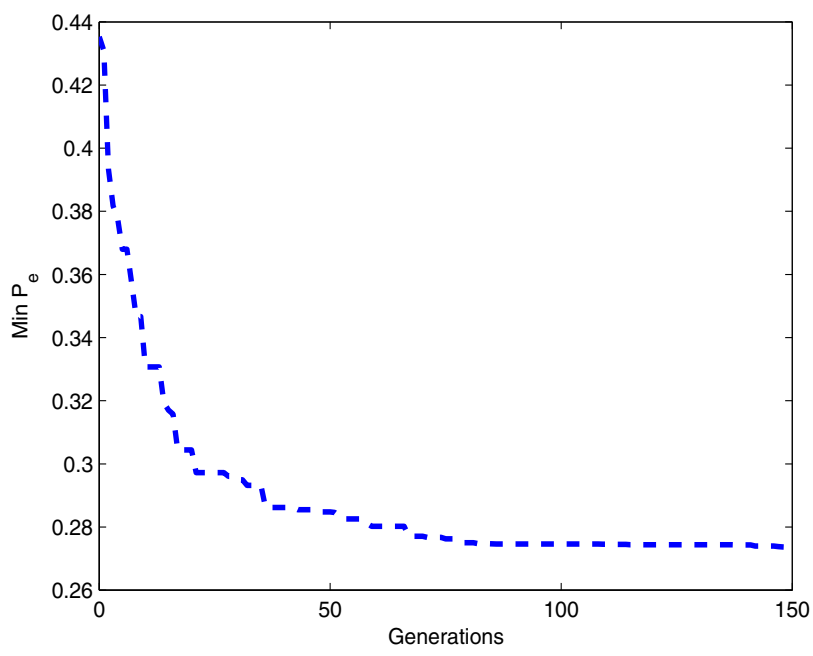

Fig. 5. Evolution of probability of error - Non-Homogenous sensors $(N=$ $3, L=4, \pi_{0}=0.5$ )

\section{CONClusion}

In this paper, we analyze the performance of a decentralized sensor network with parallel fusion architecture in the presence of correlated noise. We propose a Parallel Genetic Algorithm approach for this problem. Our results show that the optimal fusion rule for both correlated and uncorrelated observations is a majority-like fusion rule, irrespective of the degree of correlation. We also illustrate that the local decision rule plays a key role in optimizing the sensor network when the observations are correlated. If the local decisions are assumed to be LRTs and are defined completely by quantization thresholds, we show that these thresholds drift closer together as the degree of correlation increases. Finally, we demonstrate that both homogeneous and non-homogeneous sensors provide similar probability of error performance.

\begin{tabular}{|c||c|c|c||c|c|c||}
\hline \multicolumn{1}{|c||}{} & \multicolumn{3}{c||}{ Sensor 1 } & \multicolumn{3}{c||}{ Sensor 2 } \\
\hline$\rho$ & $\lambda_{1,1}$ & $\lambda_{1,2}$ & $\lambda_{1,3}$ & $\lambda_{2,1}$ & $\lambda_{2,2}$ & $\lambda_{2,3}$ \\
\hline 0 & -0.0329 & 0.2177 & 1.1636 & -0.7661 & 0.4617 & 1.6683 \\
\hline 0.2 & -0.0942 & 0.7001 & 1.6162 & -0.1284 & 0.6458 & 1.6397 \\
\hline 0.5 & -0.1513 & 0.6462 & 1.7067 & -0.1436 & 0.7152 & 1.6469 \\
\hline 0.9 & 0.1989 & 0.7523 & 1.6695 & -0.6167 & 0.5248 & 1.6571 \\
\hline
\end{tabular}

TABLE I

TABLE OF OPTIMUM LOCAL THRESHOLDS FOR DIFFERENT VALUES OF $\rho$ FOR NON-HOMOGENEOUS SENSORS CASE

\begin{tabular}{|c|c|c|c|}
\hline$\rho$ & $\lambda_{1}$ & $\lambda_{2}$ & $\lambda_{3}$ \\
\hline 0 & -0.5232 & 0.4906 & 1.3661 \\
\hline 0.2 & -0.3809 & 0.5445 & 1.3514 \\
\hline 0.5 & -0.0867 & 0.6615 & 1.2830 \\
\hline 0.9 & -0.8301 & 0.7861 & 1.2114 \\
\hline
\end{tabular}

TABLE II

TABLE OF OPTIMUM LOCAL THRESHOLDS FOR DIFFERENT VALUES OF $\rho$ FOR HOMOGENEOUS SENSORS CASE

\section{REFERENCES}

[1] J. F. Chamberland and V. V. Veeravalli, "Decentralized detection in sensor networks," IEE Transactions on Signal Processing 51, 407-416, Feb. 2003.

[2] S. Aldosari, J. Moura, "Fusion in sensor networks with communication constraints," Proc. IEEE/ACM Symposium on Information Processing in Sensor Networks (IPSN'04), Berkley, CA, 108-115, April 26-27, 2004.

[3] A. R. Reibman, "Performance and fault-tolerance of distributed detection networks," Ph.D. dissertation, Dept. Electrical Engineering, Duke Univ., Durham, NC, 1987.

[4] S. C. A. Thomopoulos, R. Viswanathan, and D. K. Bougoulias, "Optimal distributed decision fusion," IEEE Transactions on Aerospace Electronic Systems., vol. 25, pp. 761-765, Sept 1989.

[5] D. R. Halverson and G. L. Wise, "A Detection scheme for dependent noise sources," Journal of the Franklin Institute, 309, 287-300, May 1980.

[6] H. V. Poor, "Signal detection in the presence of weakly dependent noisepart I: optimum detection," IEEE Transactions on Information Theory, IT-28, 735-744, Sept 1982.

[7] G. S. Lauer and N. R. Sandell, Jr.,"Distributed detection of known signal in correlated noise," ALPHATECH, Burlington, MA, Rep. Mar. 1982.

[8] V. Aalo and R. Viswanathan, "On distributed detection with correlated sensors: two examples," IEEE Transactions on Aerospace and Electronic Systems, vol. 25, pp. 414-421, May 1989.

[9] E. Drakopolous and C. C. Lee, "Optimum multisensor fusion of correlated local decisions," IEEE Transactions on Aerospace and Electronic Systems, vol. 27, pp. 593-606, July 1991.

[10] V. Aalo and R. Viswanathan, "Asymptotic performance of a distributed detection system in correlated gaussian noise," IEEE Transactions on Signal Processing, vol. 40, pp. 211-213, Jan 1992.

[11] M. Kam, Q. Zhu and W. S. Gray, " Optimal data fusion of correlated local decisions in multiple sensor detection systems," IEEE Transactions on Aerospace and Electronic Systems, vol. 28, pp. 916-920, July 1992.

[12] J. H. Holland, Adaptation in Natural and Artificial Systems, Ann arbor University of Michigan Press, Ann Arbor, MI, 1975. 